\title{
ENHANCEMENTS TO FAILURE MODE AND EFFECTS ANALYSIS (FMEA) METHOD, AIMING TO IMPROVE RISK MANAGEMENT IN THE KNOWLEDGE- BASED ORGANIZATIONS
}

\author{
Vlad Ciprian CUPȘAN*, Mihail Aurel ȚîȚU**, Gheorghe Ioan POP** \\ *Universal Alloy Corporation Europe, Dumbrăvița, Maramureș, Romania \\ **"Lucian Blaga" University, Sibiu, Romania \\ mihail.titu@ulbsibiu.ro
}

\begin{abstract}
This paper presents the current state of risk management in the knowledge-based organisations and the importance of a preventive approach, with emphasis on the aerospace and defence industry, as well as gives detailed information on the Failure Mode and Effects Analysis (FMEA) method, in its current known state. In order to generate enhancements to the Failure Mode and Effects Analysis (FMEA) method, the strong and weak points are analysed and specific solutions are proposed for the weak points, such as occurrence scoring, with the goal of enhancing the Failure Mode and Effects Analysis (FMEA) method and, in general, of improving risk management in the knowledge-based organizations. In conclusion, the paper evaluates the improvements generated by the proposed solutions, compared to the current known method, in order to establish the value added by the enhancements.
\end{abstract}

Keywords: FMEA, risk, management, knowledge-based, organisations.

\section{Introduction}

In the current context where the competition related to the quality and delivery performance metrics between the manufacturing organisations is fierce, individual manufacturing organisations need to build on the existing knowledge and use specific tools to improve their overall performance.

In order to do so, the manufacturing organisations need to become knowledgebased organisations, by improving the general knowledge within the organisations. This can be achieved by recruiting top candidates for each area, under the scope of the organisation, as well as provide valueadding trainings to all the levels of the organisation. The third and potentially one of the most important improvements is the engagement of multi-functional teams, in all aspects where knowledge is required within the organisation.

One area in particular, which this paper is focused on, is the aerospace industry where the competition related to high quality is paramount, as lives depend on the quality levels achieved.

The aerospace components have evolved a lot in the latest decades, although the aircraft look almost the same as after the World War II. The materials used for manufacturing the aerospace components have drastically changed, as well as the shapes and thicknesses used for components, in order to reduce the costs and the weight of the aircraft.

The knowledge-based manufacturing organisations are the most affected by the changes in aerospace components due to the requirements that demand higher quality. In 
order to achieve this, some knowledgebased organisations rely on a preventive approach known as risk management.

\section{Risk management in the knowledge- based organisations}

Risk management is a state of mind within a knowledge-based organisation, which relies a lot on the following aspects:

- Management support, without which the full-scale organizational implementation is impossible. Also very important is the constant support given by management through regular risk management reviews, during which the highest risks are discussed and mitigated;

- Definition of specific risk management groups, in the case of medium to large knowledge-based organizations, where multiple product lines are used. The specific risk management groups perform the risk analysis based on the particular product requirements, process steps and functions;

- Employment of multifunctional teams. As previously stated, knowledge-based organizations need to use the multifunctional approach in order to channel all the organization's knowledge towards a specific activity. Risk management is one of the best examples where the multifunctional team approach brings benefits by employing knowledge from different relevant functions within the specific product line. Examples of functions from a manufacturing organization include:

- Production personnel;

- Quality inspectors;

- Quality engineers;

- Manufacturing engineers;

- Maintenance personnel;

- Supply chain responsible;

- Risk analysis facilitator;

- Other relevant functions.

\section{Preventive approach in aerospace and defence industry}

In the recent past, the aerospace and defence industry relied on taking preventive actions based on different types of nonstandardized analysis tools. The preventive action reports were established as derivate of the Corrective Action Report, but without including items such as containment actions, strict closure rules and in some cases, the effectiveness check.

As the moment of employment of preventive actions was difficult to be determined, it usually depended on an individual's decision.

The preventive approach in the aerospace and defence industry has changed recently by integrating a requirement related to the operational risk management in the main aerospace standard AS9100, with the release of the most recent revision, D. It includes the operational risk management as a requirement, under paragraph 8.1.1 [1]. This requirement obliges all aerospace companies, including the knowledge-based organisations, to develop, implement and control a process for risk management, with the following recommendations:

- Assignment of responsibilities for operational risk management;

- Definition of risk assessment criteria, such as: likelihood, consequences, risk acceptance;

- Identification, assessment and communication of risks throughout the operations;

- Implementation and management of actions to mitigate the risks that do not respect the acceptance criteria;

- Acceptance of the risks remaining after the implementation of actions.

In order to achieve its intended goal - to provide a preventive approach, the risk management process needs to rely on a very robust tool, which incorporates most of the recommendations identified by AS9100 D. Most of the aerospace industry companies decided to use the Failure Mode and Effects Analysis (FMEA) method. 


\section{Failure Mode and Effects Analysis (FMEA) method}

The Failure Mode and Effects Analysis (FMEA) method has its origin in the aerospace industry, more specifically in the space exploration sector, in the 1960's, during the development of the Apollo missions. The goal of the FMEA method, at that time, was to identify any safety risks that might endanger the missions and their astronauts. Once the benefits in such a high-tech sector have been observed, other industries started to develop variants of the FMEA method.

Second to NASA in using the FMEA method was the United States Navy which developed its own variant that was documented under the procedure MIL-STD $-1629$.

The third to adopt the FMEA method was the automotive industry in the late 1970s, during which the method reached a "critical mass". Due to the extent of the automotive industry it became popular in other industries. At the same time, with the usage in the automotive industry, the FMEA method expanded its scope into an area that defines it until today: product quality.

The Automotive Industry Action Group (AIAG) has defined the FMEA as a core automotive tool and has developed guidance on the method, which includes a manual "Failure Mode and Effects Analysis - FMEA Fourth Edition" [2], as well as different courses related to the FMEA implementation and usage.

The most recent large industry where FMEA has seen a revival is the same one which developed the first variant, the aerospace industry, more specifically the aircraft manufacturers and their suppliers. There are two main reasons for the revival of the FMEA method in the aerospace industry:

- The first one is the new requirement related to the operational risk management, prescribed by AS9100 D;

- The second one is the need of a proactive tool in order to reduce the costs of nonconformity.
The Failure Mode and Effects Analysis (FMEA) method is a structured brainstorming activity, with the purpose of identifying the risks related to a product, process or project. This paper will focus only on the process FMEA, which is called PFMEA. As it is a brainstorming activity, it is necessary to involve a multifunctional team, comprised of the functions within the organization.

The prerequisites to the PFMEA process are:

- The process flow;

- Product requirements;

- Process requirements.

Once the above items have been obtained, FMEA sessions can be planned. It is recommended that the session length be between 2-4 hours, as an introduction time is always needed to get the activity started.

The first step of the process FMEA activity is the documentation of the prerequisites in the FMEA form, by filling in the process step, function and requirements. The FMEA form can be documented on paper, in Microsoft Excel or in any adequate software. The second step and the first challenging brainstorming activity is to identify and document failure modes related to the product or process requirements. An example would be, if the product requirement stated, "Drill hole with diameter $10 \pm 0.5 \mathrm{~mm}$ ", one potential failure mode that can be drawn is "Hole is drilled with a diameter bigger than $10.5 \mathrm{~mm}$ ". Once the failure mode has been agreed within the FMEA team, the next step is to establish the severity of the failure mode, related to the requirement, named potential effect.This can be classified on multiple levels: end user, customer, organization, operator, process, etc. The recommendation is to document all of them, as they are used for the next step, which is scoring the severity. This is performed using a standardized scoring guide, either by a standard, such as SAE J1739 [3] or a scoring guide developed by the organization. The scores for severity range 
from 1 , being less severe, to 10 which is catastrophic. The value for the score is set by reviewing the potential effect and the scoring guide. In the case of differences of opinions within the FMEA team, the FMEA facilitator is required to negotiate the severity score. In the case of continued disagreement, the highest proposed value is selected.

The most challenging brainstorming step is the identification of the potential causes related to the potential failure mode. These are strongly linked with the knowledge available within the team and in general in the organization. The potential causes can be from areas such as Materials, Methods, Machines, Environment, Personnel, etc. and could have different levels of specificity based on the available knowledge. For each of the potential causes identified, the FMEA team needs to identify the current prevention controls related to the potential failure mode or potential cause and document them in the corresponding column. Based on the cause and prevention control, the team needs to score, from 1 to 10 , the occurrence or likelihood of the risk by using a standardized scoring guide.

The next logical step in the structure of a risk is for the FMEA team to identify the detection control, which is planned and/or implemented for the potential cause or potential failure mode. Based on the type of detection control and its location in the process, relative to the failure mode occurrence, the detection is scored by reviewing the scoring guide and using values from 1 to 10 .

Based on the scores provided for severity, occurrence or likelihood and detection, the risk priority number (RPN) is calculated by multiplication. The risk priority number (RPN) is the main criterion by which the risks are sorted and ranked, from the biggest to the smallest RPN values.

Other criteria are usually used to identify high risks, such as:

- Severity score above 9;

- Severity score above 7 and an occurrence score above 7;
- Fixed RPN thresholds.

Based on the RPN and/or other criteria, mitigation actions need to be defined for the highest risks.

\subsection{Strong points}

- The first strong point of the FMEA method is its long history and vast application areas and industries, this making the method highly documented and standardized.

- Due to its history and results, the FMEA method has already proven its value related to risk management in any organization, when applied correctly.

- The engagement of multifunctional teams for the FMEA activity in order to obtain a more robust risk analysis, by using the knowledge from different stakeholders, such as: production, quality, engineering, maintenance etc.

- The structure of the FMEA method is also a strong point due to the logical approach from requirement to potential failure mode to potential cause, which makes it easy to understand.

- The usage of scoring, from 1 to 10 for severity, occurrence and detection, which are standardized in easy to comprehend scoring guides.

- The usage of standardized scoring guides which allow the connection between, for example, the potential effect and the criteria available in the severity scoring guide (e.g. "A portion of the production lot needs to be reworked off-line" with a score of 5 for Severity). Also, between the detection control and the scoring criteria available in the detection scoring guide (e.g. "Detection in line through automated means which quantifies extend of failure mode" with a score of 5 for Detection).

- The calculation of a unique identifier of the risk importance (Risk Priority Number) relative to the other identified risks. This approach allows the prioritization of the mitigation activities based on importance and furthermore 
allows the organization to manage the risks and their effect better.

\subsection{Weak points}

- The FMEA method is dependent on a clear mapping of the processes and responsibility allocation. Due to the multifunctional approach, it is not always clear who the FMEA owner is for a given process.

- At the same time, the multifunctional team can be described as a weak point in case the organization has not defined clear rules for multifunctional team membership.

- Even in the case of complete multifunctional teams, it is possible that comprehensive knowledge is not available, due to lack of experience of the team members. In this case, the risk analysis loses value, due to potentially an incomplete understanding of the requirements or reduced knowledge related to potential causes.

- If knowledge is available, there is always the weakness related to the way the cause is documented, as causes could have different levels (as depicted by the "5 WHY" method). Thus, the FMEA multifunctional team can be unsure if the level of the cause is appropriate for mitigation.

- An important item that could potentially divert completely from the initial goal of the FMEA method, risk management, is the lack or poor definition of rules for risk mitigation. As described in the strong points paragraph, there are a lot of standards which give guidance for the FMEA method, but the lack of any agreement between the standards can lead to an incorrect usage of risk mitigation rules, such as:

- If the Risk Priority Number (RPN) is above a certain value (e.g. 100), mitigation actions need to be taken;

- All risks need to have mitigation actions;
- Different combination of high severity and high occurrence, without factoring in the detection score.

- One of the biggest weaknesses of the FMEA method is the lack of clarity related to scoring the occurrence or likelihood, especially in the cases of processes that are already implemented. The challenge for any FMEA multifunctional team to select the correct score for occurrence is given by the mixture of information:

- Actual occurrence of the failure mode;

- Actual occurrence of the cause;

- Prevention control.

\subsection{Proposed enhancements}

The below enhancements for the FMEA method also include a more robust preparation for the FMEA activity.

The first proposed enhancement is related to the standardization of the core FMEA multifunctional team, including process owner (e.g. for process FMEA, mandatory multifunctional team members should be production, quality, engineering and process owner is production).

A second enhancement is to mandate that all multifunctional team members should have at least a certain level of experience or should have undergone a certain type of training for the analysed process.

The third proposed enhancement is to document all potential causes, no matter what the level of the cause is and, in some conditions, to flow the cause to the next support process FMEA, as a potential risk. An example would be a manufacturing process FMEA, where the potential cause is defined as a specific equipment failure. This can be transferred to the Maintenance or Equipment FMEA as a potential failure mode and more detailed potential causes can be identified and mitigated, if necessary.

The fourth enhancement is to use a dedicated software solution which includes all the improvements in one place, allowing the users easy access to a standardized 
process, scoring guides, RPN automatic sorting, as well as the easy management of risks (by controlling the changes) and mitigation actions and due dates.

The fifth and most important enhancement to the FMEA method is to improve the way that the occurrence is calculated and scored, by creating a new separate score for the prevention control. This would allow the multifunctional team to score the actual or hypothetical occurrence based on the available or similar information. In addition, based on the planned or implemented prevention, to score the type of prevention control method. An example of the current method would be a risk which has a cause that is unlikely to occur or has not occurred in the analysed time interval, this would drive the occurrence score to a low value. However, the prevention control, in this example, is only a training performed for the operator, which is not the best prevention control possible. Taking into consideration that historical data is available on the risk, the nature of the prevention control is potentially ignored and only data is used for scoring.

In the enhanced version, the same risk would get a score for the occurrence based on available historical data or a score for the likelihood based on the knowledge on similar processes and a second score for the effectiveness of the prevention control. The multiplication of the Severity, Occurrence or Likelihood, Prevention, Detection scores would increase the range of potential Risk Priority Numbers (RPNs) from 1 to 10,000 , which would improve the resolution of the risk analysis. This would allow for a better management of risks and, as a novelty, would enable the organisation to manage prevention controls better and enhance the focus on the preventive approach.

\section{Conclusion}

Taking into consideration all of the above, it is fair to say that performing risk management with the current FMEA method is valuable for any knowledgebased organisation and can contribute to a significant reduction of costs of nonconforming products.

However, the current FMEA method forces the knowledge-based organisations to identify and train key employees on the FMEA method, which act as FMEA facilitators, in order to keep the multifunctional teams on track. Without the FMEA facilitator, it is very possible that the FMEA process should not be completely followed or scoring should not be performed correctly.

The enhancements proposed by this paper, if integrated into the FMEA method, would allow the removal of some key ambiguities in the current method, thus making the FMEA method more robust as a process and at the same time easier to use.

The implementation of the enhancements is possible only by using a step-by-step approach, as a lot of industries, such as the aerospace industry, are guided by the existing standards. At the same time there are areas where the usage of a specific risk analysis method is not given, thus allowing the knowledge-based organisation the choice of the method to be used, as well as the employment of new methods or enhanced methods, such the one proposed in this paper.

The risk management responsible for the knowledge-based organisation needs to evaluate the opportunity of using the enhanced FMEA method.

\section{References}

[1] Society of Automobile Engineers (SAE), Quality Management Systems - Requirements for Aviation, Space, and Defense Organizations, REV. D, https://www.sae.org, September, 2016.

[2] Automotive Industry Action Group (AIAG), Failure Mode and Effects Analysis FMEA, Fourth Edition, https://www.aiag.org, July, 2008. 
[3] Society of Automobile Engineers (SAE), Potential Failure Mode and Effects Analysis in Design (Design FMEA), Potential Failure Mode and Effects Analysis in Manufacturing and Assembly Processes (Process FMEA), https://www.sae.org, January, 2009. 